\section{NUTM1 is a recurrent fusion gene partner in B-cell precursor acute lymphoblastic leukemia associated with increased expression of genes on chromosome band 10p12.31-12.2}

For $20-25 \%$ of patients with pediatric B-cell precursor acute lymphoblastic leukemia (BCP-ALL), the driving cytogenetic aberration is unknown. Identification of the primary lesion could provide better risk stratification and even identify possible treatment options. We therefore aimed to find novel recurrent genetic aberrations in BCPALL cases. We identified an in-frame SLC12A6-NUTM1 fusion, resulting in expression of 3' exons of NUTM1, and six additional NUTM1-rearranged fusion cases. These NUTM1-rearranged cases were associated with high expression of a cluster of genes on chromosome band $10 \mathrm{p} 12.31-12.2$, including the BMI1 gene. Our data point to NUTM1 fusions as a new entity of BCP-ALL negative for known genetic abnormalities.

Pediatric BCP-ALL consists of many cytogenetic subtypes, each with a different prognosis. ${ }^{1}$ ETV6-RUNX1 fusions, high hyperdiploid, and TCF3-PBX1 fusions have a favorable prognosis, while $B C R-A B L 1$ fusions and $K M T 2 A / M L L$-rearrangements have a poor prognosis.
However, $20-25 \%$ of BCP-ALL patients do not have one of these sentinel cytogenetic aberrations and are therefore said to have B-other ALL. This B-other ALL subgroup has an intermediate risk of relapse, but includes both high- and low-risk subgroups that are currently being identified. Our laboratory identified a subtype with a similar expression profile and prognosis as $B C R-A B L 1$, namely $B C R$ - $A B L 1$-like, within the B-other ALL subgroup. ${ }^{2}$ The B-other ALL subgroup also includes other rare cytogenetic subtypes, such as intrachromosomal amplification of chromosome 21 and a dicentric chromosome $(9 ; 20) .{ }^{1}$ It is important to identify more primary lesions in the remaining B-other ALL for better risk stratification and identification of possible treatment options. In this study, we aimed to identify recurrent fusions in BCP-ALL cases without currently known lesions through RNA sequencing.

We used paired-end total RNA Illumina sequencing to detect fusion genes using STAR-fusion and FusionCatcher in a population-based ALL cohort $(n=71)$. We compared gene expression levels in a larger population-based ALL cohort $(\mathrm{n}=661)$ and an infant ALL cohort $(n=70)$ using Affymetrix U133 Plus2 expression arrays. Fluorescence in situ hybridization (FISH) was performed

Table 1. Characteristics of five pediatric and two infant NUTM1-rearranged cases.

\begin{tabular}{|c|c|c|c|c|c|c|c|c|c|c|c|}
\hline Case & Sex & Age & $\begin{array}{c}\text { WBC } \\
\text { count } \\
\left(x 10^{9} / L\right)\end{array}$ & $\begin{array}{l}\text { Protocol- } \\
\text { risk arm }\end{array}$ & $\begin{array}{c}\text { Outcome } \\
\text { (vears) }\end{array}$ & $\begin{array}{l}\text { BCP-ALL } \\
\text { subtype }\end{array}$ & Karyotype & $\begin{array}{l}\text { FISH } \\
\text { result }\end{array}$ & $\begin{array}{l}\text { NUTM1 } \\
\text { fusion }\end{array}$ & Deletions $^{1}$ & Mutations $^{2}$ \\
\hline $\begin{array}{l}1 \\
\text { (index) }\end{array}$ & M & $\begin{array}{c}14 \\
\text { (years) }\end{array}$ & 4.1 & ALL10-SR & CCR 5.0 & BCR-ABL1-like & $46, X Y[22]$ & $\begin{array}{l}\text { No break } \\
\text { apart } \\
\text { (inversion } \\
\text { between } \\
\text { probes) }\end{array}$ & $\begin{array}{c}\text { SLC12A6 } \\
\text { ex2 - NUTM1 } \\
\text { ex3 } \\
\text { (RNA-seq, RT-PCR } \\
\text { + Sanger } \\
\text { sequencing) }\end{array}$ & $\begin{array}{l}\text { Not } \\
\text { done }\end{array}$ & $\begin{array}{c}\text { FLT3 } \\
\text { p.Ile836del }\end{array}$ \\
\hline 2 & M & $\begin{array}{c}12 \\
\text { (years) }\end{array}$ & 5.4 & COALL03-HR & CCR 6.2 & B-other ALL & - & $\begin{array}{l}\text { Break } \\
\text { apart }\end{array}$ & $\begin{array}{c}\text { CUX1 ex23 - NUTM1 } \\
\text { ex5 (RNA-seq, RT-PCR } \\
+ \text { Sanger sequencing) }\end{array}$ & $R B 1$ & $\begin{array}{c}\text { No } \\
\text { mutations }\end{array}$ \\
\hline 3 & $\mathrm{~F}$ & $\begin{array}{c}8 \\
\text { (years) }\end{array}$ & 6.6 & ALL10-SR & CCR 4.8 & B-other ALL & $\begin{array}{c}\text { 46,XX, } \\
\mathrm{t}(7 ; 15)(\mathrm{q} 22 ; \mathrm{q} 1 ? 3) \\
{[9] / 46, \mathrm{XX}[1]}\end{array}$ & $\begin{array}{l}\text { Not } \\
\text { done }\end{array}$ & $\begin{array}{c}\text { CUX1 ex23 - NUTM1 } \\
\text { ex4 (RT-PCR + } \\
\text { Sanger sequencing) }\end{array}$ & $\begin{array}{c}\text { No } \\
\text { deletions }\end{array}$ & $\begin{array}{c}\text { No } \\
\text { mutations }\end{array}$ \\
\hline 5 & M & $\begin{array}{c}1 \\
\text { (years) }\end{array}$ & 48.7 & ALL8-MR & CCR 13.8 & $B C R$ - $A B L 1$-like & - & $\begin{array}{l}\text { Break } \\
\text { apart }\end{array}$ & Suspected & $\begin{array}{c}I K Z F 1 ; \\
E B F 1\end{array}$ & Not done \\
\hline
\end{tabular}

\begin{tabular}{|c|c|c|c|c|c|c|c|c|c|c|c|}
\hline 6 & M & $\begin{array}{c}6 \\
\text { (months) }\end{array}$ & 13.3 & $\begin{array}{c}\text { Interfant-99 } \\
\text { SR }\end{array}$ & CCR 9.3 & $\begin{array}{c}\text { Non-KMT2A } \\
\text { rearranged }\end{array}$ & $46, X Y[32]$ & $\begin{array}{l}\text { Not } \\
\text { done }\end{array}$ & Suspected & $\begin{array}{l}\text { Not } \\
\text { done }\end{array}$ & $\begin{array}{l}\text { Not } \\
\text { done }\end{array}$ \\
\hline
\end{tabular}

${ }^{1}$ As determined by SALSA P335 version A3 ALL-IKZF1 MLPA assay (MRC-Holland), as previously described.5 ${ }^{2}$ Custom MiSeq panel including RAS and JAK pathway genes, as previously described ${ }^{15}$ WBC: white blood cell; BCP-ALL: B-cell precursor acute lymphoblastic leukemia; FISH: fluorescence in situ hybridization; CCR, continuous complete remission; RNA-seq, RNA sequencing; ex, exon. 
A
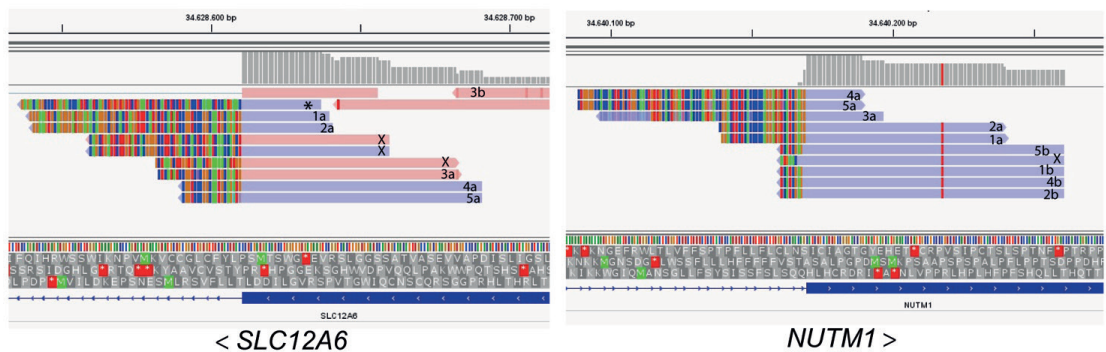

B

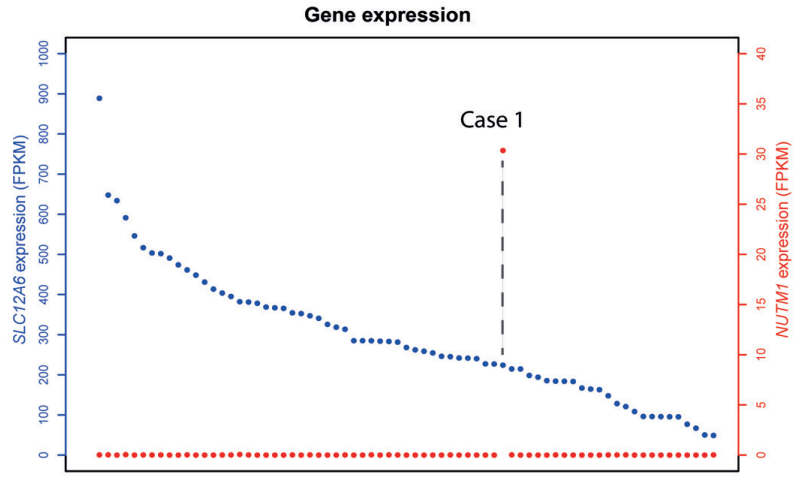

cases

C
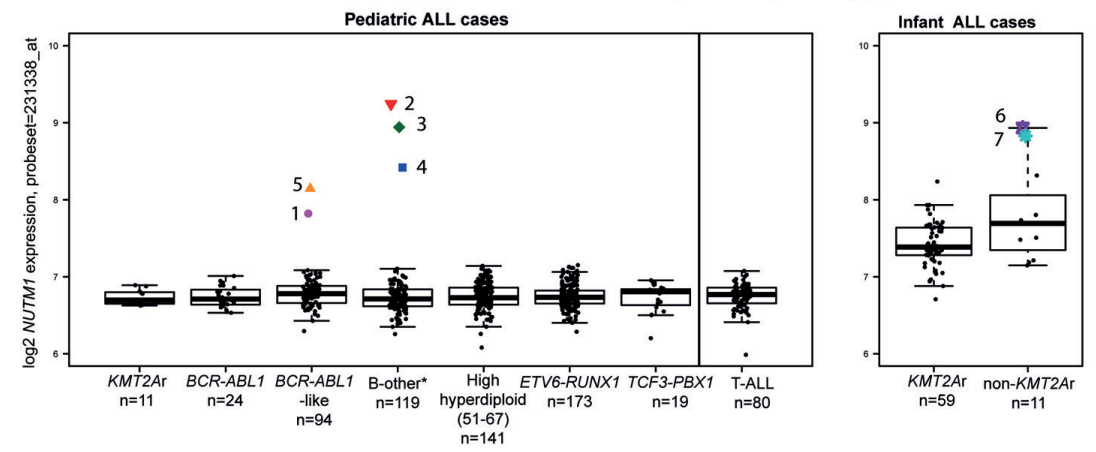

D
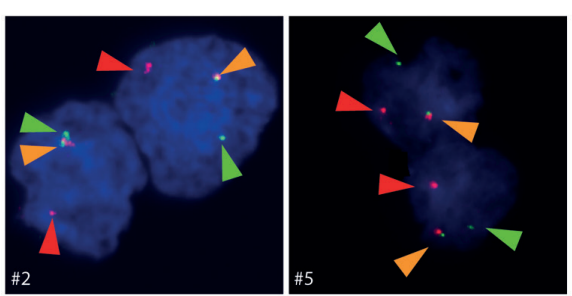

E
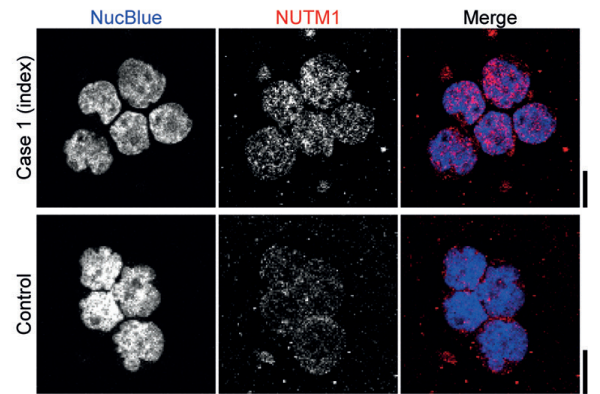

Figure 1. NUTM1 fusion identification. (A) Chimeric total RNA sequencing reads of case \#1 (SLC12A6-NUTM1 fusion case; index case) aligned with STAR and visualized with the Integrative Genomic Viewer (version 2.4.10). The bright colors (red, brown, blue, green) show mismatches with the reference genome version hg19. The left panel shows chimeric reads mapped to SLC12A6 exon 2, the right panel shows chimeric reads mapped to NUTM1 exon 3 . Matching numbers indicate that reads are from the same read pair, $a$ and $b$ indicate the two reads within a pair. * read maps to NUTM1 exon 5 , X read does not map to NUTM1. $<$ and > show directions of SLC12A6 and NUTM1. (B) Total RNA sequencing gene expression of SLC12A6 (blue) and NUTM1 (red) of all 71 cases of acute lymphoblastic leukemia (ALL), ordered on SLC12A6 gene expression in fragments per kilobase per million. The dashed line connects NUTM1 to SLC12A6 in case 1 (SLC12A6-NUTM1 fusion case; index case). (C) Boxplot showing the expression of NUTM1 (probe set 231338_at) in 661 pediatric ALL cases and 70 infant ALL cases, divided per subtype. Outliers with NUTM1 expression are displayed in color. Case \#1 is our index case shown in panel (A), cases \#2-\#7 are additionally identified by expression of NUTM1. *B-other ALL: B-cell precursor ALL without one of the sentinel aberrations (excludes KMT2A-rearranged, ETV6-RUNX1, high hyperdiploid, TCF3-PBX1, BCR-ABL1, and BCR-ABL1-like). Within the non-KMT2A-rearranged (infant) cases there are seven B-other ALL cases. KMT2Ar, KMT2A-rearranged. (D) Representative examples of fluorescence in situ hybridization (FISH) of two cases with suspected NUTM1 fusion. Orange arrows indicate a fusion signal, red arrows the break apart probe upstream of NUTM1 and green arrows the break apart probe downstream of NUTM1. Online Supplementary Figure S2 shows the FISH results of all studied cases. (E) Representative confocal microscope images of cytospins of the index case \#1 with a SLC12A6-NUTM1 fusion and a B-other ALL control sample immunostained with NUT antibody (NUTM1; red in merge). Cell nuclei were stained with NucBlue (blue in merge). Scale bars: $10 \mu \mathrm{m}$. 
using the Cytocell NUTM1 break-apart probe set MPH4800. Reverse transcriptase polymerase chain reaction (RT-PCR) was carried out using the primers shown in Online Supplementary Table S1. Immunofluorescence staining was performed using NUT antibody C52B1 (\#3625, Cell Signaling Technology). Methods are described in more detail in the Online Supplement.

RNA sequencing analysis revealed an in-frame SLC12A6-NUTM1 fusion transcript composed of exons 1-2 of SLC12A6 fused to exons 3-8 of NUTM1, encoding almost the complete coding region of NUTM1 (Figure 1A,
Online Supplementary Figure S1). This fusion was confirmed by RT-PCR and Sanger sequencing (Table 1, Online Supplementary Table S2). Both genes are located on $15 q 14$ within $5.3 \mathrm{~kb}$ distance on opposite strands, and the fusion could result from an inversion. The fusion transcript is predicted to encode a chimeric protein, likely containing an acidic binding domain for the histone acetyltransferase EP300 from NUTM1. ${ }^{3}$ The SLC12A6NUTM1 fusion case showed expression of SLC12A6 in the same range as the other BCP-ALL cases, while NUTM1 expression was high in the SLC12A6-NUTM1

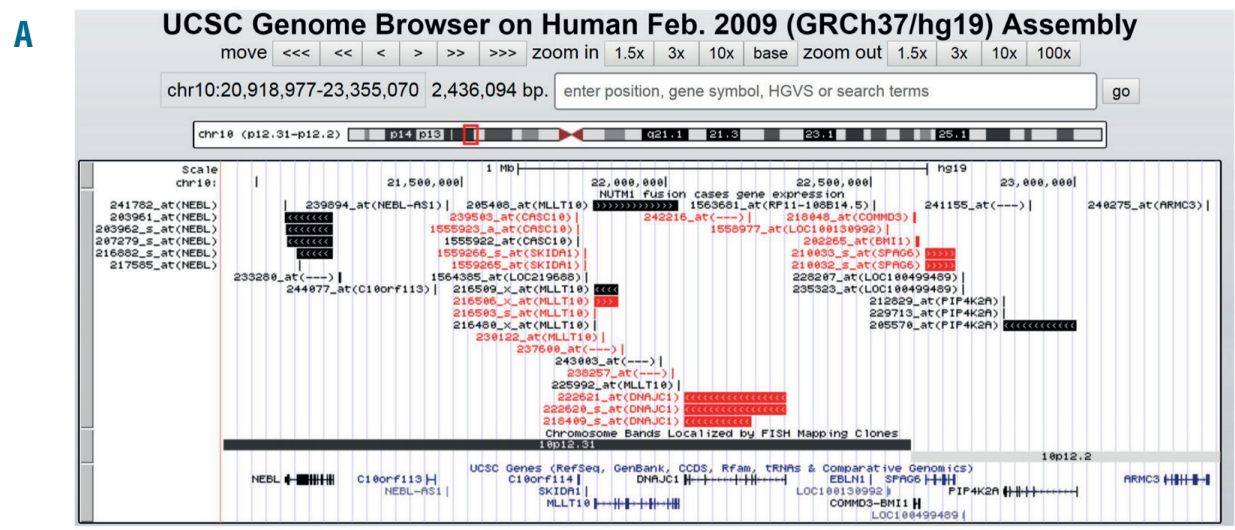

B

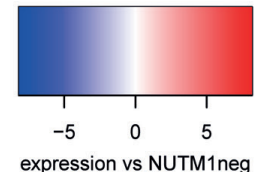

expression vs NUTM1neg

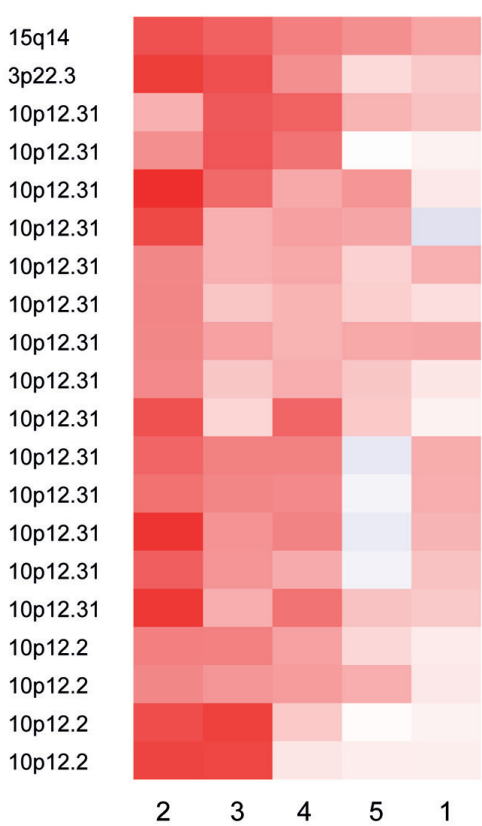

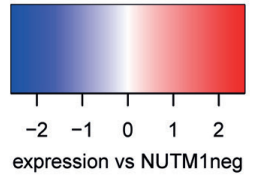

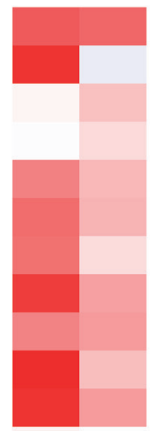

NUTM1 (231338_at) TRIM71 (231310_at) CASC10 (239503_at) CASC10 (1555923_a_at) SKIDA1 (1559266_s_at) SKIDA1 (1559265_at) MLLT10 (216503_s_at) MLLT10 (216506_x_at) MLLT10 (230122_at) --- (237600_at) --- (238257_at) DNAJC1 (222621_at) DNAJC1 (222620_s_at) DNAJC1 (218409_s_at) --- (242216_at) LOC100130992 (1558977_at) COMMD3 (218048 at) BMI1 (202265_at) SPAG6 (210033_s_at) SPAG6 (210032_s_at)

$\begin{array}{ll}6 & 7\end{array}$

Figure 2. Gene expression on chromosome band 10p12.31-12.2 in NUTM1 fusion cases. (A) Visualization of all probe sets within the 10p12.31-12.2 chromosome band. UCSC genome browser view of chromosome location (first track), probe sets (second track), chromosome band (third track), and UCSC genes (fourth track) aligned to GRCh37/hg19. In the second track, all probe sets that map to the location in this view are visualized. Each probe set is visualized with a thick band from start to end and arrows indicating the strand. A black band means no different expression between NUTM1-positive and NUTM1-negative cases, red means increased expression in NUTM1-positive cases versus NUTM1-negative cases (false discovery rate $\leq 0.01$ ). (B) Heatmap of the expression of NUTM1, TRIM71, and 10p12.31-12.2 upregulated genes in NUTM1-positive versus NUTM1-negative cases (false discovery rate $\leq 0.01$ ) in the pediatric population-based cohort. Expression of these genes is shown for the infant NUTM1-positive cases as well. Expression values are centered around 0 using the median of all Bother ALL and NUTM1 fusion cases and scaled by 2 root-mean-square for the pediatric cohort and the infant cohort individually. Blue indicates reduced expression, red indicates increased expression compared with the median over all cases per cohort. Within the 10p12.31-12.2 chromosome band, genes are ordered by genomic location. 
fusion case but absent in the remaining 70 BCP-ALL cases (Figure 1B, Online Supplementary Figure S1). NUTM1, previously known as NUT (nuclear protein in the testis), is involved in normal germ cell maturation. NUTM1 is frequently fused to BRD4 or BRD3 in NUT midline carcinoma, a subtype of squamous cell cancer, and these fusions are associated with a block in differentiation. $^{4}$

To identify additional NUTM1 fusion cases, we studied the gene expression of NUTM1 in a previously described cohort of 661 children with $\mathrm{ALL}^{5}$ and a cohort of 70 infants with ALL. ${ }^{6}$ We confirmed high expression of NUTM1 in the SLC12A6-NUTM1 fusion case (index case $\# 1$ ) and identified four additional pediatric and two infant BCP-ALL cases with high NUTM1 expression (Figure 1C, Table 1). In both cohorts, reflecting all different cytogenetic subtypes, these cases were restricted to the B-other ALL subgroup without sentinel cytogenetic abnormalities ( $\mathrm{n}=210$ pediatric, $\mathrm{n}=7$ infants). FISH with NUTM1 break apart probes could be performed for four cases with high NUTM1 expression for which cytospins were available. All four cases (three pediatric and one infant) showed a FISH break apart pattern suggesting a balanced translocation (Figure 1D, Online Supplementary Figure S2). The index case \#1 showed no FISH break apart signal, in line with a small inversion of the region between the FISH probes. RT-PCR followed by Sanger sequencing and/or RNA sequencing revealed CUX1-NUTM1 fusions involving exons $5 / 4-8$ of NUTM1 in pediatric cases \#2 and \#3 respectively, an IKZF1-NUTM1 fusion involving exons 58 of NUTM1 in pediatric case \#4, and an ACIN1-NUTM1 fusion involving exons 3-8 of NUTM1 in infant case \#7 (Table 1; Online Supplementary Table S2). Moreover, using a NUTM1 antibody, we detected nuclear staining in our index case \#1 harboring a SLC12A6-NUTM1 fusion (Figure 1E). We conclude that NUTM1 is normally not expressed in leukemic lymphoblasts and that its high level of expression in our seven patients results from a gene fusion.

Our combined results showed that NUTM1 fusions occurred in $5 / 210(2.4 \%)$ of pediatric and in $2 / 7$ of infant BCP-ALL cases without a sentinel cytogenetic aberration, and that NUTM1 has different fusion partners. Several single NUTM1 fusions were previously reported in pediatric and infant BCP-ALL. ${ }^{7-10}$ Recently, Li et al. defined a BCP-ALL expression-based subgroup in a large cohort enriched for NUTM1 fusions. ${ }^{11}$ Combining our results with the NUTM1 fusions described in literature suggests that NUTM1 fusions are a rare but recurrent event in pediatric BCP-ALL.

Among our seven NUTM1-positive cases no other recurrent deletions or mutations were detected using multiplex ligation-dependent probe amplification and a custom sequencing panel (Table 1). The recurrence of NUTM1 aberrations in BCP-ALL cases without the presence of a known driver and the resulting expression of NUTM1 suggests that NUTM1 fusions could be an oncogenic driver in leukemia. Five out of seven patients with a NUTM1 fusion were stratified into a standard-risk protocol and all seven patients are in long-term first continuous complete remission with a median follow-up time of 8.3 years (range, 4.8-13.8 years). The clinical outcomes suggest that NUTM1 fusions in BCP-ALL have a favorable prognosis. The possibly good prognosis in BCP-ALL opposes the unfavorable prognosis associated with the BRD4-NUTM1 fusion in NUT midline carcinoma; only one in 62 known patients was cured (reviewed by C.A. French). ${ }^{4}$ The apparently good prognosis of NUTM1 fusions in BCP-ALL might be due to a different role of the fusion partner or to the different cell type in which they occur.

To get an insight into the underlying biology, we compared gene expression between the five NUTM1-positive pediatric BCP-ALL cases and the remaining 112 NUTM1-negative B-other ALL [excluding BCR-ABL1-like and hypodiploid ( $\leq 39$ chromosomes)] cases and identified 130 differentially expressed probe sets (false discovery rate $\leq 0.01 ; 116$ upregulated and 14 downregulated) representing 80 unique genes (Online Supplementary Table S3). As expected, the most significant differentially expressed gene was NUTM1 (3.5-fold upregulated). The highest upregulated gene was TRIM71 (9.8-fold upregulated). Functional annotation showed enrichment of genes from chromosome bands 7p15-p14 (Bonferroni adjusted $P$-value $=9.25 \times 10^{-6}$; which harbors among others the HOXA gene cluster) and 10p12.31 $\left(P=4.05 \times 10^{-4}\right)$. Visualization of the location of differentially expressed probe sets on chromosome 10 showed that the enrichment extended to a small part of $10 \mathrm{p} 12.2$ that harbors among others BMI1 (Figure 2A). We visualized the expression of significantly differentially expressed probe sets located on 10p12.31-12.2 and 7p15-p14 in all seven NUTM1-positive cases (Figure 2B, Online Supplementary Figure S3). The genes on chromosome band 10p12.3112.2 were variably upregulated in six of seven cases, whereas the HOXA cluster was upregulated in the two highest NUTM1-expressing pediatric cases (both CUX1NUTM1) and the two infant cases (including one ACIN1NUTM1). Interestingly, Li et al. showed HOXA overexpression restricted to the same NUTM1 fusions, suggesting that upregulation of $H O X A$ genes depends on the NUTM1 fusion partner. ${ }^{11}$ In our dataset, expression of the 10p12.31-12.2 and HOXA cluster genes seems to be positively correlated to NUTM1 expression levels (Online Supplementary Figure S4). In the 10p12.31-12.2 region, expression of all genes represented on the Affymetrix U133 Plus 2 array (18 probe sets) was significantly increased in the NUTM1-positive cases (Figure 2A). In the 7p14-p15 region, expression of most but not all genes was significantly increased in the NUTM1-positive cases (Online Supplementary Figure S3B).

The NUTM1 protein is capable of binding and thereby stimulating the histone acetyltransferase activity of the EP300 protein. ${ }^{3}$ Interestingly, a single nucleotide polymorphism in chromosome band 10p12.31-12.2, specifically in an enhancer region of $B M I 1$, is associated with increased risk of BCP-ALL. ${ }^{12}$ The EP300 protein preferentially binds the risk allele of $B M I 1$ and this binding is hypothesized to increase BMII expression, resulting in leukemia via increased proliferation and reduced apoptosis. ${ }^{12}$ BMI1, a proto-oncogene, enhances self-renewal of hematopoietic stem cells and is capable of converting $B C R$ - $A B L 1$-positive progenitor cells to acute lymphoblastic leukemia. ${ }^{13,14}$ Hence, we postulate that NUTM1 fusion proteins contribute to leukemogenesis by stimulating EP300, leading to upregulation of BMI1 and other 10p12.31-12.2 genes in BCP-ALL.

In conclusion, we showed that NUTM1 rearrangement is a rare but recurrent and possible oncogenic driver event in BCP-ALL. These rearrangements seem to have a good prognosis, but this should be confirmed in larger series. The NUTM1 fusions involve many partners, resulting in overexpression of the normally silent NUTM1 gene, and are associated with upregulation of a cluster of genes on 10p12.31-12.2 including the leukemogenic BMI1 gene. 
Femke M. Hormann, ${ }^{1,2}$ Alex Q. Hoogkamer, ${ }^{1,2}$

H. Berna Beverloo, ${ }^{3}$ Aurélie Boeree, ${ }^{1,2}$ Ilse Dingjan, 1,2 Moniek M. Wattel, ${ }^{3}$ Ronald W. Stam, ${ }^{1}$ Gabriele Escherich, ${ }^{4}$ Rob Pieters, ${ }^{1,5}$ Monique L. den Boer ${ }^{1,25,6}$ and Judith M. Boer ${ }^{1,2}$

'Princess Máxima Center for Pediatric Oncology, Utrecht, the Netherlands; ${ }^{2}$ Oncode Institute, Utrecht, the Netherlands; ${ }^{3}$ Department of Clinical Genetics, Erasmus Medical Center, Rotterdam, the Netherlands; ${ }^{4}$ COALL - German Cooperative Study Group for Childhood Acute Lymphoblastic Leukemia, Hamburg, Germany; ${ }^{5}$ DCOG - Dutch Childhood Oncology Group, Utrecht, the Netherlands and ${ }^{6}$ Department of Pediatric Oncology and Hematology, Erasmus Medical Center - Sophia Children's Hospital, Rotterdam, the Netherlands

Funding: this work was supported by the Foundation Pediatric Oncology Center Rotterdam (SKOCR), the Dutch Cancer Society grant KWF-10482, and the KiKa Foundation Kika-264 grant.

Correspondence: JUDITH M. BOER.

j.m.boer-20@prinsesmaximacentrum.nl doi:10.3324/haematol.2018.206961

Information on authorship, contributions, and financial \& other disclosures was provided by the authors and is available with the online version of this article at WWW. haematologica.org.

\section{References}

1. Schwab C, Harrison CJ. Advances in B-cell precursor acute lymphoblastic leukemia genomics. HemaSphere. 2018;111(5):1.

2. Den Boer ML, van Slegtenhorst M, De Menezes RX, et al. A subtype of childhood acute lymphoblastic leukaemia with poor treatment outcome: a genome-wide classification study. Lancet Oncol. 2009;10(2):125-134.

3. Reynoird N, Schwartz BE, Delvecchio M, et al. Oncogenesis by sequestration of $\mathrm{CBP} / \mathrm{p} 300$ in transcriptionally inactive hyperacetylated chromatin domains. EMBO J. 2010;29(17):2943-2952.
4. French CA. Pathogenesis of NUT midline carcinoma. Annu Rev Pathol. 2012;7(1):247-265.

5. van $\operatorname{der}$ Veer A, Waanders E, Pieters R, et al. Independent prognostic value of BCR-ABL1-like signature and IKZF1 deletion, but not high CRLF2 expression, in children with B-cell precursor ALL. Blood. 2013;122(15):2622-2629.

6. Stam RW, Schneider P, Hagelstein JAP, et al. Gene expression profiling-based dissection of MLL translocated and MLL germline acute lymphoblastic leukemia in infants. Blood. 2010;115(14):2835-2844.

7. Andersson AK, Ma J, Wang J, et al. The landscape of somatic mutations in infant MLL-rearranged acute lymphoblastic leukemias. Nat Genet. 2015;47(4):330-337.

8. Nordlund J, Bäcklin CL, Zachariadis V, et al. DNA methylation-based subtype prediction for pediatric acute lymphoblastic leukemia. Clin Epigenetics. 2015;7(1):11.

9. Marincevic-Zuniga Y, Dahlberg J, Nilsson S, et al. Transcriptome sequencing in pediatric acute lymphoblastic leukemia identifies fusion genes associated with distinct DNA methylation profiles. J Hematol Oncol. 2017;10(1):148.

10. Lilljebjörn H, Henningsson R, Hyrenius-Wittsten A, et al. Identification of ETV6-RUNX1-like and DUX4-rearranged subtypes in paediatric B-cell precursor acute lymphoblastic leukaemia. Nat Commun. 2016;7:11790.

11. Li J, Dai Y, Lilljebjörn H, et al. Transcriptional landscape of B cell precursor acute lymphoblastic leukemia based on an international study of 1,223 cases. Proc Natl Acad Sci U S A. 2018;115(50):E11711E11720.

12. de Smith AJ, Walsh KM, Francis SS, et al. BMI1 enhancer polymorphism underlies chromosome 10p12.31 association with childhood acute lymphoblastic leukemia. Int J Cancer 2018;143(11):2647-2658.

13. Sengupta A, Ficker AM, Dunn SK, Madhu M, Cancelas JA. Bmi1 reprograms CML B-lymphoid progenitors to become B-ALL-initiating cells. Blood. 2012;119(2):494-502.

14. Rizo A, Dontje B, Vellenga E, Haan G De, Schuringa JJ. Long-term maintenance of human hematopoietic stem/progenitor cells by expression of BMI1. Blood. 2008;111(5):2621-2630.

15. Jerchel IS, Hoogkamer AQ, Ariës IM, et al. RAS pathway mutations as a predictive biomarker for treatment adaptation in pediatric B-cell precursor acute lymphoblastic leukemia. Leukemia. 2018;32(4):931940. 